\title{
Utilização de alimento industrializado por duas espécies de passeriformes (Furnarius rufus e Turdus rufiventris) em ambiente urbano
}

Use of processed foods by two passeriformes species (Furnarius rufus and Turdus rufiventris) in urban environment

\author{
D. M. Miyasaki ${ }^{1}$; E. Carrano ${ }^{2}$; M. L. Fischer ${ }^{1 *}$

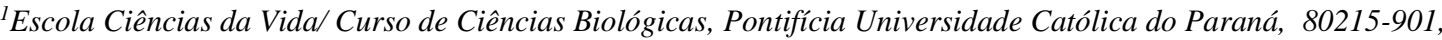 \\ Curitiba, Paraná, Brasil
}

*marta.fischer@pucpr.br

(Recebido em 9 de novembro de 2016; aceito em 11 de agosto de 2017)

\begin{abstract}
A interferência humana no comportamento de aves sinantrópicas deve ser considerada nos debates a respeito do bem-estar da fauna urbana. Assim, objetivou-se avaliar comparativamente forrageamento de Furnarius rufus e Turdus rufiventris no Campus Curitiba da Pontifícia Universidade Católica do Paraná, no período de novembro de 2012 a novembro de 2013 em duas etapas: análise do consumo parental na fase pré-oviposição e consumo de alimento na fase de cuidado parental. Os alimentos consumidos por adultos de ambas as espécies na fase pré-oviposição, se constituíram preferencialmente de insetos e restos de alimentos de diversas origens, deixados pelos frequentadores do Campus. Enquanto que na fase de cuidado parental, ambas as espécies, se alimentaram preferencialmente de alimentos industrializados, contudo, levaram ao ninho insetos e minhocas. Esse resultado evidencia que embora as aves adultas aprendam a usar recursos alternativos de maior valor energético, exibem comportamento instintivo ao alimentar os filhotes preferindo alimentos naturais, mesmo diante da alta disponibilidade de alimentos industrializados.

Palavras-chave: Aves Sinantrópicas, Bem-estar-animal, Bioética Ambiental, Ecologia Urbana, Etologia comportamental
\end{abstract}

Human interference in the behavior of synanthropic birds should be considered in debates about the welfare animal in the urban wildlife. Thus, our objective was to evaluate comparatively the foraging of Furnarius rufus and Turdus rufiventris in the Campus Curitiba of the Pontifical Catholic University of Parana in the period of November 2012 to November 2013 in two steps: analysis of parental consumption pre-oviposition and food consumption in parental care. The food consumed by adults of both species in the pre-oviposition phase is preferably compound by insects and food remains left by users of the campus. While in phase of parental care both species fed preferentially on industrialized food, however led to the nest insects and earthworms. This result shows that although adult birds learn to use alternative resources with higher energetic value, they showed instinctive behavior when feed their juveniles preferring natural foods, even in the face of high industrialized food availability.

Keywords: Animal Welfare, Behavioral Ethology Environmental Bioethics, Synanthropic Birds, Urban Ecology

\section{INTRODUÇÃO}

O Brasil abriga 1.919 espécies de aves reconhecidas pela ciência [1], sendo destas 8,3\% ameaçadas de extinção [2]. Enquanto algumas populações são mais sensíveis às alterações ambientais sofrendo declínios decorrentes do desmatamento, fragmentação, urbanização e desequilíbrio ecológico $[3,4,5,6]$, outras se adequam às novas condições ambientais. No Brasil destacam-se inúmeras espécies sinantrópicas, ou seja adaptadas ao ambiente antrópico, tais como Columbina talpacoti (Temminck 1811) (rolinha-roxa), Zenaida auriculata (Des Murs 1847) (pomba-de-bando), Furnarius rufus (Gmelin 1788) (joão-de- barro), Pitangus sulphuratus (Linnaeus 1766) (bem-te-vi), Turdus rufiventris Vieillot, 1818 (sabiá-laranjeira), Troglodytes musculus Swainson, 1831 (corruíra), além de exóticas introduzidas Columba livia Gmelin, 1789 (pombo-doméstico), Passer domesticus (Linnaeus 1758) (pardal) e Estrilda astrild (Linnaeus 1758) (bico-de-lacre). 
Dentre as aves urbanas com maior distribuição mundial e com status de espécie invasora destacam-se $C$. livia e $P$. domesticus $[7,8,9,10,11]$. A espécie Sturnus vulgaris Linnaeus, 1758 (estorninho-europeu) demanda atenção, pois embora seja considerada uma das espécies com maior capacidade de invasão do mundo [12], até o momento não foi confirmada sua ocorrência para o Brasil, requerendo ações preventivas.

O constante processo de urbanização e consequente diminuição na disponibilidade de recursos alimentares e de refúgios naturais podem impactar o comportamento até mesmo de espécies sinantrópicas, fato que se agrava diante da escassez de estudos conduzidos com aves urbanas e sua interação com a sociedade. Atualmente se destacam estudos que abordam as zoonoses e em especial relacionados à $C$. livia $[13,14]$.

O sabiá-laranjeira possui cerca de $25 \mathrm{~cm}$ de comprimento e coloração parda com ventre alaranjado. Com uma longevidade de até 30 anos em cativeiro se constitui de umas das aves territoriais e onívoras mais frequentes de áreas abertas e alteradas, sendo considerada ave símbolo do Brasil $[15,16]$. Seu ninho é constituído com fibras vegetais e barro, sendo a postura composta por três ou quatro ovos verde-azulados com pontos de sépia [16, 17]. O João-de-Barro é uma ave monogâmica, territorialista e onívora, mede de 18 a $20 \mathrm{~cm}$ de comprimento, ocorrendo do Rio Grande do Sul até o nordeste do Brasil [17, 18]. Os ninhos são construídos a cada estação reprodutiva, geralmente em galhos de árvores e em edificações, compostos por barro, fibras vegetais, estrume e pedregulhos. A postura compreende de dois a quatro ovos [17, 18, 19]. Apesar de ambas as espécies serem habituais e abundantes nos centros urbanos, estudos biológicos e etológicos são escassos, principalmente sobre T. rufiventris. No Brasil as espécies são referidas em estudos sobre levantamentos faunísticos [6, 19], ecológicos [5, 20,21] e etológicos [15, 18, 22, 23, 24].

A suplementação alimentar para aves é uma conduta cultural praticada globalmente que vem desencadeando a atenção de inúmeras pesquisas que visam aviar o impacto na mesma a curto e longo prazo nas populações de aves urbanas [25]. Segundo Jones e Reymond (2008) [25], disponibilizar alimentos atraem as aves aos espaços urbanos proporcionando interação com a biodiversidade de uma maneira ética contrapondo à tradicional prática de manter os pássaros cativos. A suplementação alimentar é importante principalmente em locais com invernos rigorosos e consequente escassez de recursos, contudo, mesmo diante dos benefícios atestados no sucesso reprodutivo, Jones e Reymond (2008) [25] alertaram para necessidade de estudos locais que atestem impactos como na disseminação de doenças, risco de gerar dependência e influência de uma alimentação pobre em aves nas fases de pré-oviposição e de oviposição. Embora a convivência com os humanos possa desencadear alterações comportamentais em diversas aves, resultando em declínios ou aumentos populacionais, poucos são os estudos que abordam essa temática $[14,26]$, fato que justifica o presente estudo. Assim, questiona-se se há alteração no comportamento alimentar de aves adultas e filhotes em decorrência da disponibilidade de alimentos industrializados. Desta forma, objetivou-se avaliar comparativamente o forrageamento de $F$. rufus e T. rufiventris durante o período reprodutivo (fase de pré-oviposição e cuidado parental) no Campus da Pontifícia Universidade Católica do Paraná na cidade de Curitiba, Paraná.

\section{MATERIAL E MÉTODOS}

\section{1 Área de estudo}

O presente estudo foi realizado no Campus da Pontifícia Universidade Católica do Paraná ( $25^{\circ} 27^{\prime} 02.35^{\prime}$ 'S e $40^{\circ} 15^{\prime} 10.23^{\prime}$ 'W), na cidade de Curitiba, Paraná, Brasil (Figura 1), no período de novembro de 2012 a novembro de 2013, em duas etapas: análise do consumo alimentar parental na fase pré-oviposição e na fase de cuidado parental. A seleção do local para estudo baseou-se em parâmetros como disponibilidade de diferentes sítios para nidificação, distintas fontes alimentares e a existência de um intenso fluxo de pessoas geradoras de resíduos alimentares.

Localizado no Bairro do Prado Velho, o Campus da PUCPR é um ambiente urbanizado cercado por prédios, residências, ruas e avenidas. Possui área aproximada de $67.000 \mathrm{~m}^{2}$ constituída predominantemente por edificações, estacionamentos e jardins, onde circulam diariamente cerca 
de 30.000 pessoas. A composição florística é formada por espécies ornamentais e paisagísticas, destacando-se: palmeira-rabo-de-peixe (Caryota sp, Arecaceae), jerivá (Syagrus romanzoffiana, Arecaceae), caliandra-rosa (Calliandra brevipes, Fabaceae), paineira (Ceiba speciosa, Malvaceae), jacatirão (Tibouchina pulchra, Melastomataceae) e, ainda, uma vegetação arbórea secundária ou com espécies exóticas nas margens do rio Belém, que atravessa a universidade [28].

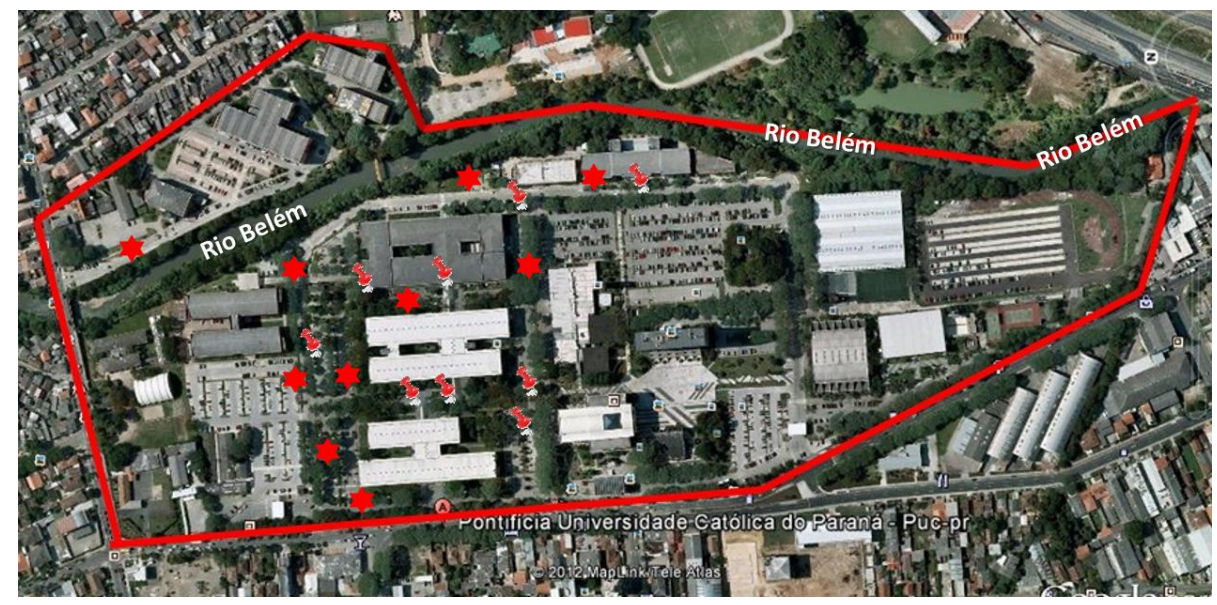

Figura 1. Imagem aérea do Campus da Pontifícia Universidade Católica do Paraná limitada pela linha vermelha, com destaque para o percurso do rio Belém e os pontos de observação dos ninhos de F. rufus e T. rufiventris

Fonte: Modificado de Google Earth.

\subsection{Análise do consumo parental na fase pré-oviposição}

As observações foram desenvolvidas através da aplicação do método animal focal [29], cujo registro do forrageamento de aves adultas de $F$. rufus e $T$. rufiventris visou identificar o tipo de alimento consumido e sua frequência. As observações foram realizadas três vezes por semana, durante duas horas, sendo destinada uma hora para cada espécie, no período da manhã das 9 horas às 11 horas da manhã, durante os meses de setembro e outubro de 2013. Foram categorizados os alimentos (naturais e industrializados) consumidos e o local de forrageio. $\mathrm{O}$ esforço amostral totalizou 24 horas de observação.

\subsection{Consumo de alimento na fase de Cuidado Parental}

Na segunda etapa foi verificado se $F$. rufus e T. rufiventris entravam no ninho portando resíduos de alimentos industrializados deixados por frequentadores e estudantes do Campus. Para tal, foram aplicados dois métodos, o primeiro de observação direta e outro de experimentação. Para observação direta, foram catalogados os alimentos consumidos pelos adultos e os levados pelos mesmos até o ninho. Inicialmente, foi necessário mapear os ninhos presentes no Campus e posteriormente selecionados dez ninhos de cada espécie. O critério de seleção foi à localização do ninho a uma altura viável para visualização e acessibilidade. Durante as observações, foi verificado se os ninhos possuíam ovos ou filhotes, o tempo de permanência dos pais dentro e fora do ninho à procura de alimentos e o tipo de alimento levado até o ninho.

Para a experimentação foram oferecidos, em uma bandeja plástica $(9,0 \times 30,0 \times 49,0 \mathrm{~cm})$ camuflada (com folhas verdes e terra), quatro itens alimentares contendo: a) frutas (todas constituintes do repertório alimentar das espécies e disponíveis em uma mesma quantidade e tamanho similar de cinco unidades/pedaços): pitanga (Eugenia uniflora, Myrtaceae), mamão (Carica papaya, Caricaceae) e amora-preta (Morus nigra, Moraceae), b) larvas de tenébrios (Tenebrio molitor), c) alimentos industrializados à base de glúten (e.g. pão e salgadinho extrusado a base de milho). Justifica-se a escolha destes itens, pois os mesmos foram os mais frequentes em 
um levantamento prévio de recursos alimentares disponíveis em áreas externas, estacionamentos e jardins do Campus.

A bandeja foi colocada equidistante, cerca de três metros do ninho e do observador. Durante as observações dos ninhos as visitas à bandeja foram acompanhadas registrando-se os exemplares de $F$. rufus e T. rufiventris presentes no Campus, o item alimentar recolhido da bandeja. Aplicouse o método de observação animal focal e análise de todas as ocorrências, sendo que o tempo de monitoramento para cada ninho foi de 50 minutos. Ambos os métodos, observação e experimentação, foram realizados simultaneamente durante todo o período de reprodução, no turno da manhã entre 6 horas e 12 horas e 30min, três vezes por semana, sendo que cada ninho foi monitorado por pelo menos duas vezes na semana, alternando-se as espécies, totalizando 43 horas e 30 minutos.

\section{Procedimentos Estatísticos e legais}

Para análise da homogeneidade dos dados de frequência relativa, tais como itens consumidos nas fases reprodutivas e entre as espécies, utilizou-se o teste de qui-quadrado considerando como hipótese nula a inexistência de diferenças entre as amostras. Para os dados de média, tais como consumo de cada item, tempo dispendido e média de entrada e saída do ninho, utilizou-se os testes não paramétricos Kruskall-Wallis e Mann-Whitney, considerando a significância de 95\%. O presente estudo foi aprovado pelo Comitê de Ética no Uso de Animais da Pontifícia Universidade Católica do Paraná (parecer $\left.n^{\circ} .749\right)$ e possui autorização do SISBIO ( ${ }^{\circ}$ 39037-1).

\section{RESULTADOS}

Na fase de pré-oviposição, a frequência de consumo dos itens alimentares por adultos de $F$. rufus e $T$. rufiventris ocorreu na mesma proporção, sendo que insetos e alimentos industrializados foram mais consumidos quando comparados com frutas e minhocas (Figura 2). A frequência relativa ao consumo de cada item diferiu entre as espécies $\left(\mathcal{X}^{2}(3)=258 ; \mathrm{p}<0,001\right)$, sendo que a ingestão de frutas como pitanga (E. uniflora) e mamão (C. papaya) e minhocas foi significativamente mais frequente para T. rufiventris (Figura 2). Durante o período reprodutivo, foram mapeados 96 ninhos (ativos e inativos) de T. rufiventris $(\mathrm{N}=32)$ e $F$. rufus $(\mathrm{N}=64)$. A análise dos 10 ninhos selecionados resultou na constatação de diferenças significativas nos itens levados ao ninho, predominando insetos em ambos (Figura 2). Para T. rufiventris houve um aumento significativo no consumo de frutos e minhoca e diminuição de industrializados $\left(x_{(3)}^{2}=120\right.$, $\mathrm{P}<0,001)$ e para $F$. rufus diminuição significativa do consumo de industrializados e aumento de consumo de minhocas $\left(x^{2}{ }_{(3)}=2566, \mathrm{P}<0,001\right) \mathrm{O}$ carregamento de frutas foi registrado apenas para T. rufiventris. A média de alimentos industrializados levados ao ninho foi menor do que de insetos e minhocas (Tabela 1).

Tabela 1. Média ( \pm desvio padrão; amostra; mínimo-máximo) dos itens alimentares levados aos ninhos por Furnarius rufus $e$ Turdus rufiventris.

\begin{tabular}{lcc}
\hline Item consumido & $\begin{array}{c}\text { Furnarius rufus } \\
\left(\varkappa^{2}(3)=2 ; \mathrm{p}<0,0001\right)\end{array}$ & $\begin{array}{c}\text { Turdus rufiventris } \\
\left(\varkappa^{2}(3)=53 ; p<0,0001\right)\end{array}$ \\
\hline Insetos & $4,8 \pm 2,8(\mathrm{~N}=26 ;$ i.v. $=0-12) \mathrm{Aa}$ & $3,7 \pm 1,8(\mathrm{~N}=26 ;$ i.v. $=0-7) \mathrm{Aa}$ \\
Frutas & $0 \pm 0(\mathrm{~N}=26 ;$ i.v. $=0-0) \mathrm{Ca}$ & $0,7 \pm 1,2(\mathrm{~N}=26 ;$ i.v. $=0-4) \mathrm{Cb}$ \\
Minhocas & $1,9 \pm 0,8(\mathrm{~N}=26 ;$ i.v. $=0-4) \mathrm{Ba}$ & $1,7 \pm 0,9(\mathrm{~N}=26 ;$ i.v. $=0-4) \mathrm{Ba}$ \\
Industrializados & $0,1 \pm 0.6(\mathrm{~N}=26 ;$ i.v. $=0-3) \mathrm{Ca}$ & $0,2 \pm 0,4(\mathrm{~N}=26 ;$ i.v. $=0-1) \mathrm{Ca}$ \\
\hline
\end{tabular}

Os valores absolutos foram comparados através do teste Kruskall-Wallis, sendo os valores significativamente diferentes representados por letras distintas. As letras maiúsculas correspondem à comparação dos itens em cada uma das espécies, enquanto as letras minúsculas correspondem à comparação entre as duas espécies. 


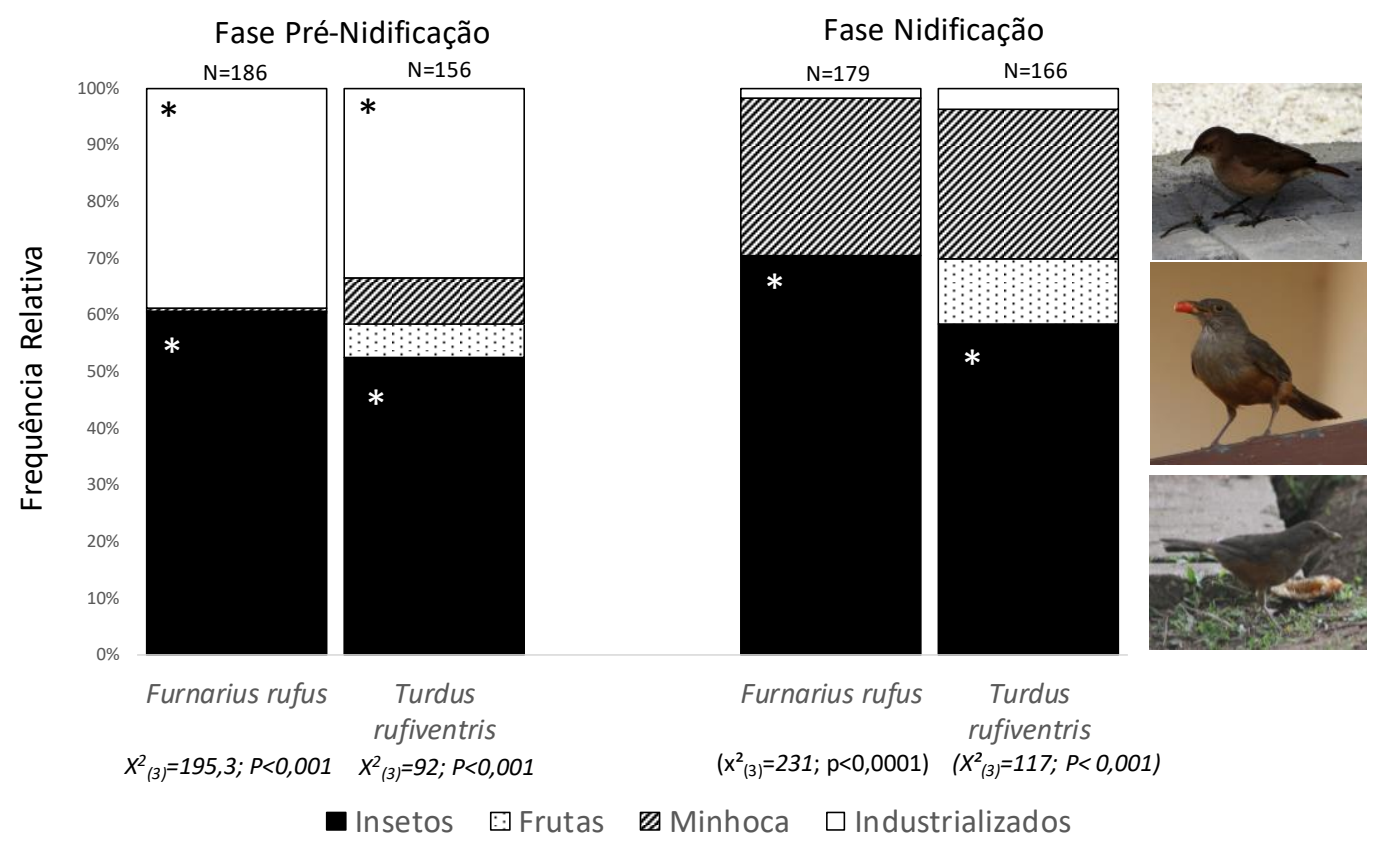

Figura 2. Frequência relativa dos itens alimentares minhoca, insetos, frutas e industrializados (restos de alimentos) consumidos na fase pré-oviposição e levados ao ninho na fase de nidificação por adultos de Furnarius rufus $e$ Turdus rufiventris. Os valores absolutos foram comparados através do teste do quiquadrado, sendo as diferenças significativamente maiores $(P<0,05)$ acompanhadas de asterisco $(*)$. As imagens de cima para baixo são referentes a Furnarius rufus alimentando-se de libélula (Odonata); $\mathrm{T}$. rufiventris alimentando-se de um fruto de Eugenia uniflora (pitanga) $e \mathrm{~T}$. rufiventris consumindo alimento industrializado. Fonte: Cátia de Paula Sant'Anna e Dayane Mayumi Miyasaki.

Durante as visitas das aves à bandeja, $F$. rufus e T. rufiventris alimentaram-se no local, sendo que a frequência dos itens consumidos na bandeja pelas espécies diferiu $\left(\varkappa^{2}(3)=53 ; p<0,0001\right.$; $x^{2}(3)=2 ; \mathrm{p}<0,0001$, respectivamente). Para T. rufiventris, o consumo de salgadinho foi maior do que de pão, frutas e larvas de tenébrio. Já para $F$. rufus, salgadinho e tenébrio foram os itens mais consumidos (Figura 3).

Constatou-se que a frequência dos itens levados da bandeja ao ninho divergiu para $T$. rufiventris $\left(\mathcal{x}^{2}(3)=22 ; \mathrm{p}<0,0001\right)$ e para $F$. rufus $\left(\mathcal{x}^{2}(3)=82 ; \mathrm{p}<0,0001\right)$, pois embora ambas as espécies tenham optado mais por larvas de tenébrio, o consumo de frutas foi registrado apenas para T. rufiventris e a alimentação do parceiro apenas em $T$. rufiventris com larvas de tenébrio (Figura $3)$. 


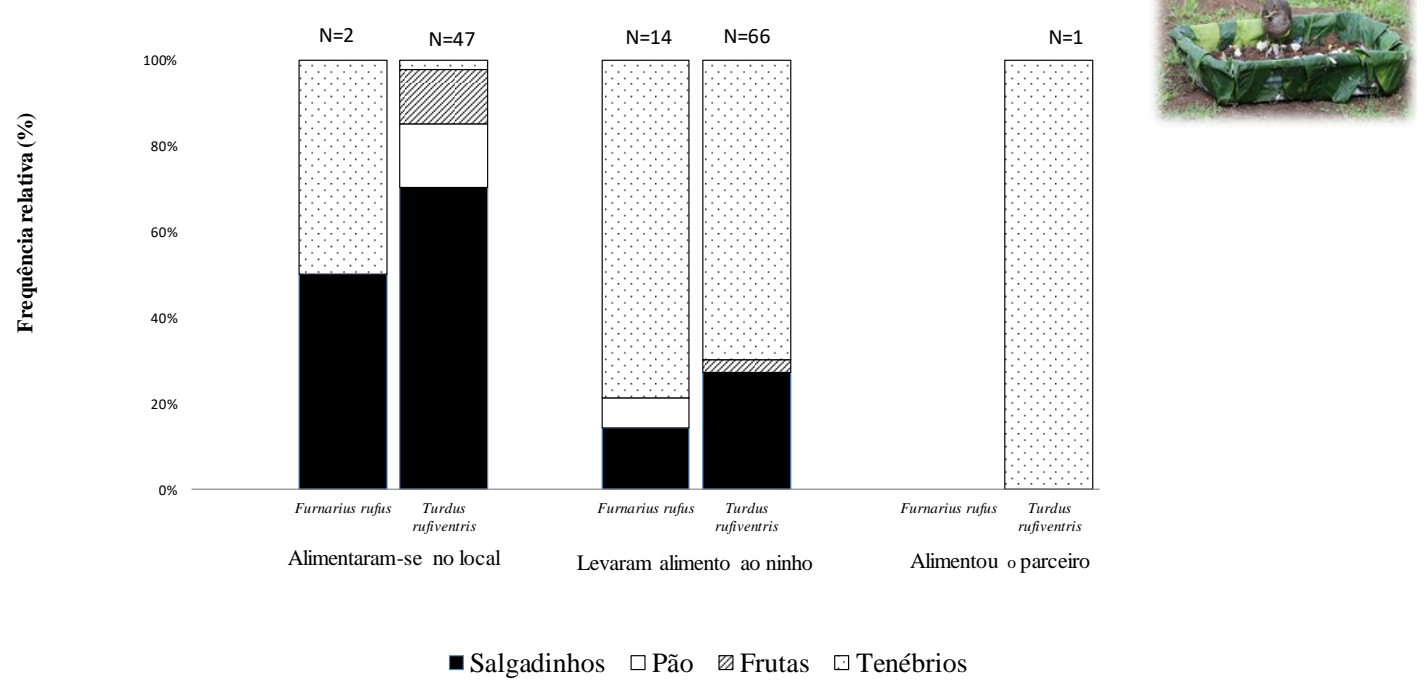

Figura 3. Frequência relativa dos itens alimentares quando $\mathrm{F}$. rufus $e \mathrm{~T}$. rufiventris alimentavam-se na bandeja, levavam alimento ao ninho ou alimentavam parceiro. Imagem: Exemplar de Turdus rufiventris capturando larvas de tenébrio em bandeja utilizada para o experimento. Fonte. Dayane Mayumi Miyasaki

O número médio de entradas e saídas do ninho foi maior para $T$. rufiventris, entretanto o número médio de entradas ao ninho com alimentos foi maior para $F$. rufus (Tabela 2). Constatouse que adultos de ambas as espécies permaneceram mais tempo fora do que dentro dos ninhos, porém quando comparado entre as espécies, $T$. rufiventris permaneceu por mais tempo fora do ninho (Tabela 2).

Tabela 2. Média (desvio padrão; amostra; mínimo-máximo) do número de entradas, saídas e entrada com alimento no ninho e tempo médio de permanência fora e dentro do ninho por Furnarius rufus $e$ Turdus rufiventris.

\begin{tabular}{lcc}
\hline Parâmetros & Furnarius rufus & Turdus rufiventris \\
\hline Entradas ao ninho & $11 \pm 6,7(\mathrm{~N}=26 ;$ i.v.=1-28) a & $8,5 \pm 3,4(\mathrm{~N}=26 ;$ i.v.=4-17) a \\
Saídas do ninho & $12 \pm 6,4(\mathrm{~N}=26 ;$ i.v.=1-28) a & $8,8 \pm 3,2(\mathrm{~N}=26 ;$ i.v.=4-18) a \\
Entradas no ninho com alimento & $6,8 \pm 3,1(\mathrm{~N}=26 ;$ i.v.=1-15) a & $6,4 \pm 2,3(\mathrm{~N}=26 ;$ i.v. $=1-12) \mathrm{b}$ \\
Tempo de permanência fora do & $168,3 \pm 201(296 ; 3-1980)$ a & $207,2 \pm 171,6(230 ; 4-915) \mathrm{b}$ \\
ninho (seg) & $70 \pm 142(286 ; 1-1200)$ a & $85,7 \pm 144,9(220 ; 1-1453) \mathrm{a}$ \\
$\begin{array}{l}\text { Tempo de permanência dentro do } \\
\text { ninho (seg) }\end{array}$ & \\
\hline
\end{tabular}

\section{DISCUSSÃO}

Os dados do presente estudo contribuem para uma compreensão mais expressiva das adaptações na ocupação de ambientes urbanos por aves sinantrópicas. O maior consumo de insetos e alimentos industrializados durante a fase pré-oviposição por $F$. rufus e de T. rufiventris, provavelmente está relacionado a dois fatores: à disponibilidade e distribuição dos recursos alimentares e a seletividade destas espécies por itens mais energéticos, inerentes às demandas metabólicas durante o período reprodutivo.

Embora a área de estudo apresente disponibilidade de insetos, inerente aos ambientes urbanos [28], destacou-se pela disponibilidade de alimentos industrializados decorrente do descarte proveniente do expressivo montante de frequentadores [28]. O processo de urbanização e 
fragmentação de hábitats influencia diretamente na disponibilidade e diversidade de recursos alimentares [6, 30, 31]. Seja devido ao fato das áreas verdes urbanas, em geral, apresentarem pouca complexidade estrutural e diversidade, sendo constituídas principalmente por espécies exóticas [32]. Ou mesmo pela demanda de alimentos artificiais, os quais em situações mais deliberadas são diretamente oferecidos pelo homem com intuito de aproximar-se dos animais [25, 33].

A seletividade por itens alimentares mais energéticos na fase de pré-oviposição e cuidado parental é uma estratégia esperada $[4,19,25]$. Insetos e alimentos industrializados se constituem de uma rica fonte de lipídios, ácidos graxos, carboidratos e proteínas [37], e aparentemente, as aves são hábeis em diferenciar a composição nutricional, escolhendo os itens que suprem com maior eficiência suas necessidades como constatado para Tangara episcopus (Linnaeus 1766) [38] e em aves migratórias [39].

No caso de $F$. rufus e $T$. rufiventris os insetos podem oferecer altos teores de proteínas, equivalendo à contribuição da proteína animal para os seres humanos [37], enquanto que carboidratos, além das frutas, podem ser obtidos em grandes quantidades de restos de alimentos consumidos pelos humanos. Sendo assim, provavelmente o elevado consumo de restos alimentares registrados no presente estudo representaria uma maneira eficaz de agregar nutrientes à dieta, sem grandes demandas de energia em competição por itens naturais mais escassos. Pierotti e Annett (1991) [40] e Auman et al. (2008) [41] atestaram que embora as aves que consomem alimentos industrializados sejam mais pesadas, a sua dieta é nutricionalmente mais pobre, fato que pode acarretar em problemas na saúde do animal comprometendo seu bem-estar a curto, a médio e longo prazo, a estrutura da população e da comunidade [25].

$\mathrm{O}$ fato de $F$. rufus e $T$. rufiventris levarem predominantemente insetos e minhocas para o ninho ao invés de alimentos industrializados, aumenta a expectativa da relação nutricional com cuidado parental e reforça a suposição que sejam hábeis em diferenciar a composição dos recursos alimentares [38]. Segundo Schwagmeyer e Mock (2008) [42], em muitas espécies, os ninhegos recebem uma dieta de maior qualidade do que aquelas usadas por adultos para sua própria manutenção. Enquanto o consumo de alimentos industrializados está relacionado ao aprendizado, o cuidado parental reflete um comportamento instintivo. Assim, acredita-se que o consumo de alimentos industrializados como pão, salgadinho, pipoca e arroz, implicaria em um processo de aprendizado baseado em tentativa-e-erro maximizado pelo processo de urbanização e aumento da disponibilidade destes itens alimentares [43], corroborando com as evidências de Argel-de-Oliveira et al. (1998) [44] para Pitangus sulphuratus.

Enquanto que, a o ato predominante de entrar no ninho com insetos e minhocas parece refletir um padrão comportamental que aparece na forma funcional completa na primeira vez em que é executado, mesmo que o animal não tenha experiência prévia com os estímulos que provocam tal comportamento. Neste sentido, possivelmente as aves instintivamente sejam capazes de diferenciar os alimentos que fazem parte de sua dieta natural, dos alimentos artificiais, rejeitandoos ou oferecendo com menor frequência aos filhotes, uma vez que, provavelmente, esses alimentos não fazem parte da dieta natural da espécie.

Embora ambas as espécies apresentem dieta diversificada e generalista, o fato das frutas terem sido levadas ao ninho somente por T. rufiventris, reflete o hábito natural da espécie em incluir esse item à sua dieta, tanto em ambientes urbanos quanto em ambientes naturais [15,45], quando comparado com $F$. rufus, a qual apresenta um hábito alimentar predominantemente insetívoro.

A interpretação quanto ao comportamento alimentar discrepante entre as espécies deve considerar o grupo funcional e a guilda trófica na qual $F$. rufus se caracteriza como uma espécie insetívora de áreas abertas $[46,47]$ que forrageia quase que exclusivamente ao nível do solo. Já $T$. rufiventris possui maior plasticidade alimentar sendo classificado como onívoro, consumindo frutos e insetos [15,45] forrageando em bordas e interior de ambientes florestados, tanto ao nível do solo quanto em diferentes estratos arbóreos.

$\mathrm{O}$ fato de $F$. rufus dispender menos tempo para retornar ao ninho e apresentar um maior número de entradas e saídas do ninho do que observado para $T$. rufiventris, subsidia a evidência de que possuem mais facilidade para encontrar os alimentos preferenciais a serem levados aos ninhegos. Embora T. rufiventris tenha levado frutas aos seus filhotes, ressalta-se que a área de estudo apresenta baixa riqueza e abundância de espécies frutíferas. Sendo assim, T. rufiventris teria que buscar estes frutos em outros locais mais distantes de seus ninhos, ou até mesmo fora do 
Campus, justificando o registro do menor número de entradas no ninho com alimentos e do maior tempo de permanência fora dos mesmos, corroborando com os estudos de Manhães (2003) [48], Loss e Silva (2005) [49] e Pascotto (2006) [50].

Tanto a propensão pelo consumo de alimentos industrializados pelas aves adultas durante a fase pré-oviposição quanto à opção de levar preferencialmente alimentos naturais ao ninho, foi confirmado através da experimentação com a bandeja camuflada. A experimentação foi uma forma de simular quais itens alimentares as aves adultas escolheriam para se alimentar, ou levar ao ninho, quando todos os itens estivessem reunidos, em uma mesma quantidade e em um mesmo substrato diferente daqueles presentes no Campus, os quais já são conhecidos e correlacionados com a presença de alimentos.

$\mathrm{O}$ fato de $F$. rufus ter consumido elevadas quantidades de alimentos industrializados e menor variedade de outros itens quando comparado com $T$. rufiventris, sugere relação com a distância de fuga. Percebeu-se durante as observações, assim como no cotidiano, que $F$. rufus se aproxima com maior facilidade e aparentemente não demonstra atitudes aversivas com relação às pessoas, viabilizando a aquisição de restos de alimentos oriundos do consumo humano. Ao contrário, a suscetibilidade à aproximação por humanos demonstrada por T. rufiventris inibe o recolhimento dos recursos alternativos, demandando de outras fontes nutricionais como frutas e minhocas. De fato, pesquisadores como Feninger (1983) [34] têm constatado que a distância de fuga de algumas aves como $C$. livia, $P$. domesticus e, principalmente, F. rufus diminuíram, propiciando a circulação em áreas urbanas entre milhares de pessoas e de veículos. Em situações mais extremas essa alteração comportamental pode, inclusive, potencializar chances de atropelamentos, principalmente por ampliarem o horário e o local de forrageamento diante da diminuição da oferta de alimentos [35]. A diferença da distância de fuga entre as espécies estuadas obviamente reflete na maior variedade de itens consumidos por $T$. rufiventris em relação a $F$. rufus, corroborando com os registros de Vogel (2014) [36] para T. rufiventris os quais podem maximizar o consumo dos itens alimentares de maior disponibilidade.

\section{CONCLUSÃO}

Os resultados do presente estudo vêm a somar com o conhecimento a respeito da ecologia urbana atestando o uso do recurso alimentar proveniente dos seres-humanos por aves adultas de $T$. rufiventris e $F$. rufus. O Presente estudo aponta que aves adultas embora consumam alimentos industrializados, todavia optam por prover seus ninhegos com alimentos naturais, sugerindo a possibilidade de reconhecimento das propriedades nutricionais do alimento. Contudo, deve-se considerar o impacto de uma dieta artificial e desequilibrada a curto prazo no bem-estar do animal, acrescido da potencialidade de desencadeando doenças, as quais a longo prazo podem impactar na estrutura da população e da comunidade constituinte da fauna urbana. A demanda por novos paradigmas éticos na interação com os animais revoga práticas tradicionais de aprisioná-los com a justificativa de que as pessoas têm o direito de conviver com outras espécies e vivenciar a prazerosa experiência do controle de suas vidas. A prática de suplementação alimentar de espécies não domesticadas, como aves urbanas, é uma forma promover o convívio e cuidado das pessoas com os animais. Ressalta-se que é fundamental que haja um monitoramento dos alimentos ofertados e os riscos associados com a transmissão de doenças, geração de dependência, promoção de deficiência nutricional e maximização da reprodução de espécies invasoras. Tal resultado é um alerta para as políticas de conservação e manejo da fauna urbana que visem o bem-estar das mesmas, uma vez que o excesso de alimentos artificiais pode impactar na saúde dos animais. Desta forma, esse tema deve fazer parte dos debates intermediados pela Bioética Ambiental, que visa equalizar os argumentos e valores de cada ator envolvido nessa questão a fim de se alcançar uma solução que abarque valores comuns visando a sustentabilidade e o bem-estar de todos os seres envolvidos, desta e de futuras gerações. 


\section{AGRADECIMENTOS}

Agradecemos aos estagiários do Laboratório Núcleo de Estudos do Comportamento Animal, do Curso de Ciências Biológicas da PUCPR, em especial à a Cátia de Paula Sant'anna, João Selesbram e Brunna Victorino.

\section{REFERÊNCIAS BIBLIOGRÁFICAS}

1. Piacentini VQ, Aleixo A, Agne CE, Maurício GN, Pacheco JF, Bravo GA, Brito GRR, Naka LN, Olmos F, Posso S, Silveira LF, Betini GS, Carrano E, Franz I, Lees AC, Lima LM, Pioli D, Schunck F, Amaral FR, Bencke GA, Cohn-Haft M, Figueiredo LFA, Straube FC, Cesari E. Annotated checklist of the birds of Brazil by the Brazilian Ornithological Records Committee / Lista comentada das aves do Brasil pelo Comitê Brasileiro de Registros Ornitológicos. Rev Bras Ornitol. 2015;23(2):91-298.

2. IUCN - International Union for Conservation of Nature 2014. The IUCN Red List of Threatened Species. Version 2014.1.<http://www.iucnredlist.org >. [Acesso em 25/02/2014].

3. Piratelli A, Sousa SD, Corrêa JS, Andrade VA, Ribeiro RY, Avelar LH, Oliveira EF. Busca por bioindicadores de fragmentação florestal: aves Passeriformes na Floresta Atlântica do Sudeste do Brasil. Braz J biol. 2008;68(2):259-268.

4. Piratelli A, Pereira MR. Dieta de aves na região leste de Mato Grosso do Sul, Brasil. Ararajuba 2002;10(2):131-139.

5. Pereira PZ, Pereira PM, Arantes AC, Melo C. Monitoramento de ninhos de aves em um parque urbano. Rev Bras Zoociênc. 2009;11(1):39-45.

6. Cruz BB, Piratelli AJ. Avifauna associada a um trecho urbano do Rio Sorocaba, Sudeste do Brasil. Biota neotrop. 2011;11(4):255-264.

7. Johnson MS, Pluck H, Hutton M, Moore G. Accumulation and renal effects of lead in urban populations of feral pigeons, Colum balivia. Environ Contam Toxicol. 1982;11:761-767.

8. Sacchi R, Gentilli A, Razzetti E, Barbieri F. Effects of building features on density and flock distribution of feral pigeons Columba livia var. domestica in an urban environment. Can J Zool. 2002;80(1):48-54.

9. Swaileh KM, Sansur R. Monitoring urban heavy metal pollution using the House Sparrow (Passer domesticus). J Environ Monit. 2005;8:209-213.

10. Ferman LM, Peter HU, Montalti D. A study of feral pigeon Columba livia var. in urban and suburban areas in the city of Jena, Germany. Arxius de Miscellània Zoològica. 2010;8:1-8.

11. Scain G. Prevalência de Cryptococcus neoformans em fezes de pombos (Columba livia) nas praças públicas da cidade de Lages, Santa Catarina [monografia] Universidade do Extremo Sul Catarinense UNESC; 2011, 39 p.

12. Lowe S, Browne M, Boudjelas S, Poorter M. 100 the world's worst invasive alien species. Aliens. 2000;12:1-12.

13. Nunes VFP. Pombos urbanos: o desafio de controle. Rio de Janeiro. Revista Carioca de Controle de Pragas Urbanas, Vetores e Pragas. 2003;65:89-92.

14. Amâncio S, De Souza BV, Melo C. Columba livia e Pitangus sulphuratus como indicadoras de qualidade ambiental em área urbana. Rev bras ornitol. 2008;16(1):32-37.

15. Gasperin G, Pizo MA. Frugivory and habitat use by thrushes (Turdus spp.) in a suburban area in south Brazil. Urban Ecost. 2009;12(4):425-436.

16. Lepage D. AviBase: the world bird database. BirdLife International.<http://avibase.bsceoc.org/avibase.jsp?lang=EN>. 2012. [Acesso em: 12/07/2013].

17. Sick H. Ornitologia Brasileira. Rio de Janeiro: Nova Fronteira; 1997. 912 p.

18. Tiepolo LM, Costa LCM. Estudo do comportamento de Furnarius rufus Gmelin, 1788 (Aves Passeriformes - Furnariidae) em Curitiba, Paraná, Brasil [monografia] Pontifícia Universidade Católica do Paraná, Curitiba; 1994, 50 p.

19. Braga VT. O investimento em cuidado parental é igual entre os sexos em uma espécie monogâmica Furnarius rufus? [dissertação] Programa de Pós-Graduação em Ecologia e Conservação, Universidade Federal Do Paraná, Curitiba, PR, Brasil. 25/08/2013); 2012, 36 p.

20. Ricklefs RE. Comparative Demography of New World Populations of Thrushes (Turdus spp.). Ecol Monograph. 1997;67:23-43.

21. Toledo BCM, Donatelli JR, Batista TG. Relation between green spaces and bird community structure in an urban area in Southeast Brazil. Urban Ecos. 2012;15:111-131.

22. Efe AM, Filippini A. Nidificação do joão-de-barro, Furnarius rufus (Passeriformes, Furnariidae) em estruturas de distribuição de energia elétrica em Santa Catarina. Ornithologia. 2006;1(2):121-124. 
23. Marreis TI, Sander M. Preferência ocupacional de ninhos de joão-de-barro (Furnarius rufus, Gmelin) entre área urbanizada e natural. Biodiv Pampeana. 2006;4:29-31.

24. Souza LF, Santos AC. Climate and nest opening orientation in Furnarius rufus (Furnariidae). Iheringia, Sér Zool. 2007;97:293-295.

25. Jones DN, Reynolds S. Feeding birds in our towns and cities: a global research opportunity. J Avian Biol. 2008;39(3):265-271

26. Torga K, Franchin GA, Júnior MO. A avifauna em uma seção da área urbana de Uberlândia, MG. Biotemas. 2007;20(1):7-17.

27. Pontifícia Universidade Católica do Paraná (PUC-PR), A Universidade. <http://www.pucpr.br/institucional/campus/index.php>. 2014 [Acesso em15/02/2014].

28. Falk JH. Energetics of a suburban lawn ecosystem. Ecology 1976;141-150.

29. Del-Claro K, Prezoto F. Comportamento animal. Uma introdução à Ecologia Comportamental. Jundiaí: Livraria Conceito; 2004, 132 p.

30. Fávaro FDL, Anjos LD. Microhabitat of Habia rubica (Vieillot) and Trichothraupis melanops (Vieillot) (Aves, Emberizidae, Thraupinae) in an Atlantic Forest, Southern Brazil. Rev bras Zool. 2005;22(1):213217.

31. Marques CP. Psitacídeos (Aves: Psittaciformes) em praças de Uberlândia, MG: um estudo sobre a exploração de recursos no ambiente urbano [dissertação] Universidade Federal de Uberlândia, Minas Gerais, MG; 2012, 45 p.

32. Gomes MAS, Soares BR. A vegetação nos centros urbanos: considerações sobre os espaços verdes em cidades médias brasileiras. Estudos Geográficos. 2003;1(1):29-39.

33. O'Leary R, Jones DN. The use of supplementary foods by Australian magpies Gymnorhina tibicen: implications for wildlife feeding in suburban environments. Austral Ecol. 2006;31(2):208-216.

34. Feninger O. Estudios cuantitativos sobre aves em areas urbanas de Buenos Aires con densa poblacion humana. Hornero no. Extraordinário. 1983;174-191.

35. Souza SG. Fatores influentes sobre atropelamentos de vertebrados silvestres em rodovias da região central do estado do Rio Grande do Sul, Brasil [dissertação] Pós-graduação em Biodiversidade animal, Universidade Federal de Santa Maria, Rio Grande do Sul, Brasil. 2010; 65p.

36. Vogel HF, Metri R, Zawadzki CH. Comunidade e partilha ecológica de turdídeos (Aves: Passeriformes) em um fragmento urbano de floresta com araucárias em Guarapuava no Sul do Brasil. Arq ciênc vet zool UNIPAR. 2014;17(2):149-150.

37. Manzano-Agugliaro F, Sanchez-Muros MJ, Barroso FG, Martínez-Sánchez A, Rojo S, Pérez-Bañón C. Insects for biodiesel production. Renewable and Sustainable Energy Reviews. 2012;16(6):3744-3753.

38. Bosque C, Calchi R. Food choice by blue-gray tanagers in relation to protein content. Comp Biochem Phys A. 2003;135(2):321-327.

39. Bairlein F, Gwinner E. Nutritional mechanisms and temporal control of migratory energy accumulation in birds. Annu Rev Nutr. 1994;14(1):187-215.

40. Pierotti R, Annett CA. Diet choice in the herring gull: constraints imposed by reproductive and ecological factors. Ecology. 1991;319-328.

41. Auman HJ, Meathrel CE, Richardson A. Supersize me: does anthropogenic food change the body condition of Silver Gulls? A comparison between urbanized and remote, non-urbanized areas. Waterbirds. 2008;31(1):122-126.

42. Schwagmeyer PL, Mock DW. Parental provisioning and offspring fitness: size matters. Ani Behav. 2008;75(1):291-298.

43. Resende BD, Ottoni EB. Brincadeira e aprendizagem do uso de ferramentas em macacos-prego (Cebusapella). Estudos de psicol. 2002;7(1):173-180.

44. Argel-de-Oliveira MM, Curi NA, Passerini, T. Alimentação de um filhote de Bem-te-vi, Pitangus sulphuratus (Linnaeus) (Passeriformes, Tyrannidae), em um ambiente urbano. Rev Bras Zool. 1998;15(4):1103 -1109.

45. Del Hoyo J, Elliot A, Christie DA. Handbook of Birds of the World. vol. 10. Cuckoo-shrikes to trushes. Barcelona: Lynx Ediciones; 2005. 895 p.

46. Willis EO. The composition of avian communities in remanescent woodlots in southern Brazil. 1979.

47. Carrano E. Efeitos da fragmentação e perturbação sobre aves de remanescentes de Floresta Ombrófila Mista no estado do Paraná [dissertação]. Programa de Pós-Graduação em Ecologia e Conservação Universidade Federal do Paraná; 2013. 167 p.

48. Manhães MA. Variação sazonal da dieta e do comportamento alimentar de traupíneos (Passeriformes: Emberizidae) em Ibitipoca, Minas Gerais, Brasil. Ararajuba. 2003;11(1).

49. Loss ACC, Silva AG. Comportamento de forrageio de aves nectarívoras de Santa Teresa-ES. Natureza on line. $2005 ; 3(2): 48-52$ 
50. Pascotto MC. Avifauna dispersora de sementes de Alchornea glandulosa (Euphorbiaceae) em uma área de mata ciliar no estado de São Paulo. Rev bras ornitol. 2006;14(3):291-296.

51. Wilson E O. Biophilia, the human bond with other species, Harvard University Press, Cambridge, Mass; 1984. 